\title{
ADS-B Like UTM Surveillance Using APRS Infrastructure
}

\author{
Ying-Hong Lin ${ }^{1,2}$, Chin E. Lin ${ }^{1, *}$ and Hsu-Chan Chen ${ }^{2}$ \\ 1 UAV Center, Chang Jung Christian University, Tainan 711301, Taiwan; inhon@mail.cjcu.edu.tw \\ 2 School of Information and Design, Chang Jung Christian University, Tainan 711301, Taiwan; \\ sspsjin11tw@gmail.com \\ * Correspondence: chinelin@mail.cjcu.edu.tw; Tel.: +886-987071071
}

Received: 29 April 2020; Accepted: 18 July 2020; Published: 21 July 2020

\begin{abstract}
Automatic packet reporting system (APRS) is selected as a candidate for automatic dependent surveillance-broadcast (ADS-B) like solution for unmanned aircraft system traffic management (UTM). The APRS on-board unit (OBU) at $0.5 \mathrm{~W}$ radio power and a proper ground transceiver station (GTS) deployment together makes up the infrastructure for unmanned aerial vehicle (UAV) surveillance. The airborne APRS OBU, designed and built using an available LightAPRS module, and the GTS to relay received data into the UTM Cloud is developed in this study. By system integration, the APRS OBU reports position data and flight data periodically to UTM Cloud. This paper presents the development of the ADS-B like operation for UTM using APRS. To avoid communication jamming by HAMs, the adopted APRS shifts its carrying frequency from 144.64 MHz to $144.61 \mathrm{MHz}$. In addition, the data period is tuned to around $10 \mathrm{~s}$ to test its functional capability. The APRS OBU downlinks 90 bytes of surveillance data onto the UTM cloud using the Internet, including position and flight data from Pixhawk flight controller (FC). A series of system performance verifications are conducted to examine APRS ADS-B like reliability and continuity with coverage limit. Through 19 flight tests, the GTS collected 1330 packets of data out of 1331 transmitted from the APRS OBU. Each data packet has the complete 90 bytes for surveillance with position and six degree-of-freedom (DoF) flight data on the UTM cloud. The APRS GTS deployment, with a low rate of missing data, covers a $40 \mathrm{~km}$ radius at the specific frequency of $144.61 \mathrm{MHz}$. The test results verify the functional capability of APRS to support an ADS-B like for UTM in Taiwan.
\end{abstract}

Keywords: UAV; UTM; ADS-B like; APRS; system performance

\section{Introduction}

The CNS/ATM project by the International Civil Aviation Organization (ICAO) increased air traffic management (ATM) performance in the National Airspace System (NAS) by using the satellite technologies of global positioning system (GPS) and satellite communication (SATCOM) for seamless operation [1]. Under this system infrastructure and environment, the automatic dependent surveillance (ADS) was developed to enforce aircraft surveillance by broadcasting its position data to aircraft and ground ATC centers in its vicinity. ADS-B affiliates airborne aircraft for clear traffic awareness.

For UAV development, the unmanned aircraft system traffic management (UTM) proposed by the National Air and Space Administration (NASA) constructs a feasible and effective system to assist UAV operation for aviation safety [2]. The UTM system is applied to small UAVs in low altitudes and to large mission UAVs into the integrated airspace. From the ATC prospective, UAVs going into integrated airspace becomes a great concern to NAS flight safety [3]. Using the same concept from ATM, a hierarchical UTM system is proposed for construction in Taiwan for civil UAVs [4]. 
ADS-B has been proven to be effective and efficient in aviation surveillance. It is very important both in ATM as well as in UTM. Since the traffic alert and collision avoidance system (TCAS) have become a mature standard, the active detect and avoid (DAA) mechanism enables collision avoidance from UAVs and manned aircraft to enhance aviation safety [5]. Referring to the ADS-B system concept, an ADS-B like surveillance system is proposed with four feasible systems [6]. TCAS for ATM or DAA for UTM may be executed by software to estimate the closest point of approach (CPA) between two approaching aircraft and generate warning signals for avoidance [7]. The ADS-B TCAS concept used in the air transportation system can be extended into ADS-B like DAA in the UAVs.

In the developing UTM, communication downlink from air to ground is a key solution for UAV surveillance. UAV communication can use plenty of frequency resources in military systems, but it is limited in civil systems. UAVs may be classified by their weight and thrust into small or large scale UAVs with rotor wings and fixed wings. Command and control (C2) [8] is the main function for operating visual line-of-sight (VLOS) or beyond visual line of sight (BVLOS) flight. Small UAVs (sUAVs) will be legal to fly below 400 feet in urban to remote areas for a variety of applications. UAVs can be included into UTM to be transparent to ATM. For those high performance long endurance fixed wing UAVs, their operation will definitely threaten NAS flights. UTM will need to merge into the integrated airspace in high altitude with ATM [2,9]. The UTM shall link to ATM via ADS-R [10,11].

The ADS-B like communication infrastructure is proposed by introducing 4G/LTE (long term evolution), LoRa (long range wide area network) and APRS (automatic packet reporting system) for small UAVs [6]. In UTM development [2], the airspace of 400 feet altitude is regularly controlled for small UAVs at lower speeds. In Taiwan, UAVs flying below 400 feet is authorized for local government for management, while those above 400 feet is controlled and monitored by Civil Aeronautical Administration (CAA) [4,9]. A hierarchical UTM is constructed under such segmentation to establish regional UTM (RUTM) and national UTM (NUTM) for prompt management [4].

The proposed hierarchical UTM tries to construct a prototype for the demonstration of the UAV surveillance and collision avoidance system based on the constructed ADS-B like communication infrastructure [6]. Orrell et al. presented an ADS-B like system concept using 4G/LTE for the small UAV (sUAV) surveillance in low altitude [12]. Unlike manned aircraft, since sUAV are not detectable via radar or other independent surveillance techniques, the dependent surveillance mechanism shall be feasible to develop. Mobile communication is the most affordable communication system to adopt for its opening to the public in most areas. 4G/LTE is available to use with UAVs. However, 4G/LTE cannot be guaranteed for those flying above 400 feet. Based on the ADS-B like system concept, the construction of a UAV surveillance infrastructure using multiple communication media is proposed [4,6]. In addition to 4G/LTE, LoRa, APRS, and XBee are eligible candidates for ADS-B like for UAV [6]. The ADS-B like candidates have different characteristics compared in Table 1.

Ramsey introduced a small ADS-B device by "uavionix" [13] for UAVs. It uses commercial frequency $1090 \mathrm{MHz}$ and $978 \mathrm{MHz}$ on UAVs. However, radio stations for UAV ADS-B are not designed to provide coverage down to low altitudes [12]. The UAV service providers are not affordable for the construction of a new infrastructure. Scardina presented the use of ADS-B for the general categories of commercial air transportation systems [14] by FAA and ICAO. Under very limited ADS-B authorizations, it is difficult to broadly open to all types of UAVs, especially those small ones flying below 400 feet.

In this paper, the APRS as an ADS-B like candidate for UTM is well developed. The APRS on-board unit (OBU) is designed and built with an aim towards low-cost, light-weight, high-reliability, and wide-coverage through ground transceiver station (GTS) deployment. APRS GTS is deployed to establish communication gateways to relay radio surveillance data from UAVs onto the UTM cloud. In the preliminary surveys, the APRS GTS covers a $40 \mathrm{~km}$ radius range or longer. The OBU design and GTS deployment for APRS, by system integration with proper firmware programming, is the core technology to downlink the message to UTM surveillance. 
APRS OBU fabrication, firmware programming, and system integration are discussed in detail in this paper. UAV flight tests from different test sites are demonstrated to show the effect of APRS OBU performance on UTM [15]. APRS OBU will be an important redundant system for the complete ADS-B Like solution in UTM.

Table 1. ADS-B Like characteristics comparison.

\begin{tabular}{|c|c|c|c|c|}
\hline Device & 4G/LTE & LoRa & X-Bee & APRS \\
\hline Character & Public & \multicolumn{3}{|c|}{ Professional } \\
\hline Coverage & Except remote & \multicolumn{3}{|c|}{ 0-25 miles into air } \\
\hline Range and Altitude & $<500$ feet $A G L$ & \multicolumn{3}{|c|}{$<5000$ feet AGL } \\
\hline Relay Sta. & Exist & \multicolumn{3}{|c|}{ Need to add } \\
\hline Price & $>650$ US & \multicolumn{3}{|c|}{$<100$ US } \\
\hline System & Available & \multicolumn{3}{|c|}{ Need construction 1000-1500 US/each } \\
\hline Data rate & Video & \multicolumn{3}{|c|}{ Data } \\
\hline Weight & $<300 \mathrm{~g}$ & \multicolumn{3}{|c|}{$<200 \mathrm{~g}$} \\
\hline Voltage & \multicolumn{4}{|c|}{$5-12 \mathrm{Vdc}$} \\
\hline Power & \multicolumn{4}{|c|}{$<1 \mathrm{~W}$} \\
\hline Cloud & Direct & \multicolumn{3}{|c|}{ Relay onto via Gateways } \\
\hline Legal radio & \multicolumn{2}{|c|}{ Legal, authorize to system providers } & \multicolumn{2}{|c|}{ Legal, need application } \\
\hline Frequency by NCC regulation & $902-928 \mathrm{MHz}$ & $920-925 \mathrm{MHz}$ & $\begin{array}{c}920-928 \mathrm{MHz} \\
2.4 \mathrm{GHz}\end{array}$ & $144-146 \mathrm{MHz}$ \\
\hline Technique for UTM & \multicolumn{2}{|c|}{ Mature and ready } & \multicolumn{2}{|c|}{ Need license and setting } \\
\hline
\end{tabular}

\section{Materials and Methods}

The proposed hierarchical UTM [4] requires a surveillance system infrastructure including ground infrastructure and OBU for UAVs. The regional UTM (RUTM) and national UTM (NUTM) shown in Figure 1 are proposed for UAV surveillance management under local government and CAA in Taiwan [9]. The APRS has the widest coverage range of the proposed ADS-B like solutions for UTM.

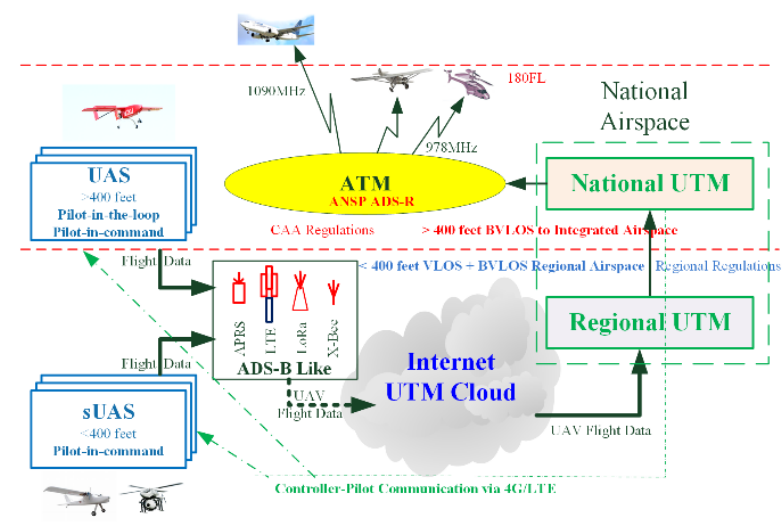

Figure 1. The hierarchical UTM under construction in Taiwan $[4,11]$.

The APRS is commonly used by HAMs and volunteer rescue groups. In most Asian countries, a specific frequency of $144.64 \mathrm{MHz}$ is assigned for public use. The transceiver station for APRS is called i-Gate. The APRS i-Gates relay messages via the Internet to any destination. There are more than $200 \mathrm{i}-G$ ates distributed over Taiwan. It is very convenient to use APRS as an ADS-B Like solution for UAVs. Since an APRS at $144.64 \mathrm{MHz}$ is noisy from many irregular users, to avoid communication traffic jamming, the UTM project has applied a public specific frequency of $144.61 \mathrm{MHz}$ for UAV 
OBUs. The reason for selecting $144.61 \mathrm{MHz}$ is to avoid frequent conflict from other users. From our preliminary tests [6], each i-Gate uses the $6 \mathrm{dBi}$ antenna can cover a $40 \mathrm{~km}$ radius range operation for an APRS with $0.5 \mathrm{~W}$ radio power. It is particularly useful for high altitude fixed wing UAVs flying into remote and mountain territories.

In the hierarchical UTM development, the APRS OBU is designed and fabricated using a 'LightAPRS' module [16] to integrate with Pixhawk flight controller (FC) [17] for UAVs. The ground transceiver station (GTS) is designed with an off-the-shelf KENWOOD TM721G radio frequency receiver, "TNC-pi" [18] (terminal node controller) and Raspberry pi to relay messages onto the Internet to the UTM Cloud database. Figure 2 shows the proposed ADS-B Like system structure using APRS. The TNC-pi demodulates audio signal into digital signal, and then passes it to Raspberry Pi (RPi), a single board computer with Linux OS, for further processing and storing onto the UTM Cloud. The UTM cloud is constructed with a database to manipulate all UAV surveillance data to regional UTM (RUTM) operation centers [4].

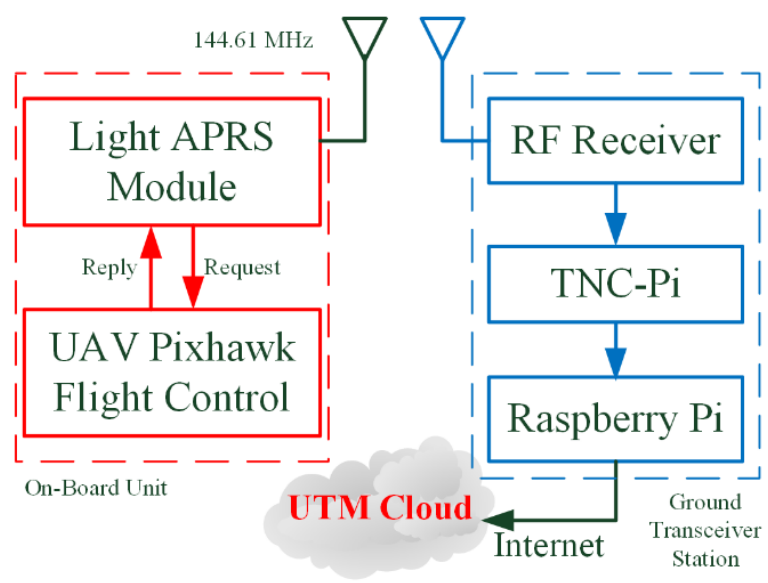

Figure 2. ADS-B like system structure using APRS.

\subsection{APRS On-Board Unit (OBU)}

The APRS OBU is fabricated with a LightAPRS module with a specific firmware design shown in Figure 3. The firmware starts the process of assigning the UAV ID, pilot ID, audio frequency, call-sign SSID, and symbol code. The ADS-B Like surveillance data format is specified including: [Heading(5); UAV_ID(6); Pilot_ID(6); Lat.(9); Long.(10); Alt.(4); $6 \mathrm{DoF}(\mathrm{Vx}, \mathrm{Vy}, \mathrm{Vz}, \alpha, \beta, \gamma)$ (36); V(6); A(6); Tail(2)]. The Heading and Tail in surveillance data are for distinguishing the transmitter from the other HAM users. The ADS-B Like surveillance data not only contains position tracking but also includes 6 DoF flight control data at 90 Bytes. The data format is designed with the purpose of collecting UAV flight performance into Big Data for further study and analysis [4,6]. The original data ending with the UAV operation current is collected and arranged in AX.25 format [19]. Then, the digital data is modulated into audio frequency using frequency shift keying (AFSK) for transmission by the built-in RF module in LightAPRS at $144.61 \mathrm{MHz}$, which is distinguished from HAM users at $144.64 \mathrm{MHz}$.

The data format of the ADS-B Like solution for UTM surveillance is shown in Table 2. For APRS OBUs, the flight control data $\left(\mathrm{V}_{\mathrm{x}}, \mathrm{V}_{\mathrm{y}}, \mathrm{V}_{\mathrm{z}}, \alpha, \beta, \gamma\right.$, voltage, current) are from the Pixhawk FC, and the position data (latitude, longitude, and altitude) are from a built-in GPS module in LightAPRS. Note that the three-axis flight speed $V_{x}, V_{y}$, and $V_{z}$ are based on the inertial north-east-down (NED) frame. The UART port is used for the connection between LightAPRS and Pixhawk FC, and the MAVLink protocol [20] is used to communicate with the Ardupilot flight control software in the Pixhawk for requesting flight data and setting the time interval. 


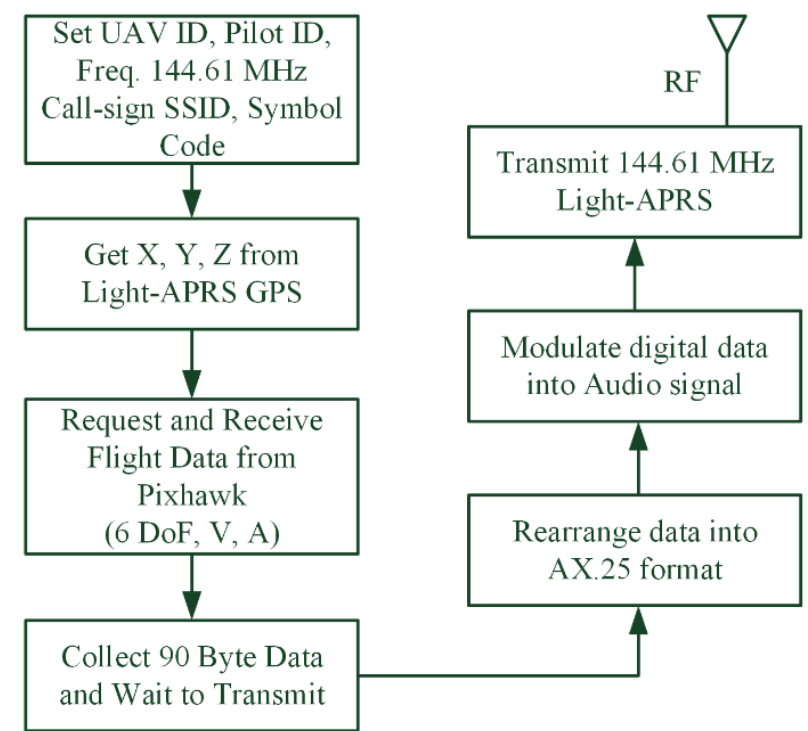

Figure 3. Firmware flowchart of APRS OBU.

Table 2. ADS-B Like surveillance data format.

\begin{tabular}{|c|c|c|c|}
\hline Data & Bytes & Data & Bytes \\
\hline Heading & 5 & Tailing & 2 \\
\hline UAV ID & 6 & Pitch $\alpha$ & 6 \\
\hline Pilot ID & 6 & Yaw $\beta$ & 6 \\
\hline Latitude & 9 & $\operatorname{Roll} \gamma$ & 6 \\
\hline Longitude & 10 & Voltage & 6 \\
\hline Altitude & 4 & Current & 6 \\
\hline $\mathrm{V}_{\mathrm{x}}$ & 6 & $\mathrm{~V}_{\mathrm{z}}$ & 6 \\
\hline $\mathrm{V}_{\mathrm{y}}$ & 6 & & \\
\hline \multicolumn{4}{|c|}{ Data unit and format } \\
\hline Data & Unit & & \\
\hline Latitude & deg. & $\begin{array}{r}x x . x) \\
\text { e.g., } 22\end{array}$ & \\
\hline Longitude & deg. & $\begin{array}{r}x x x . x \\
\text { e.g., } 12\end{array}$ & \\
\hline Altitude & feet & $\begin{array}{r}\text { Int } \\
\text { e.g. }\end{array}$ & \\
\hline$V_{x}, V_{y}, V_{z}$ & $\mathrm{~m} / \mathrm{s}$ & $\begin{array}{r}x x \\
\text { e.g. }\end{array}$ & \\
\hline$\alpha, \beta, \gamma$ & deg. & $\begin{array}{r}\text { Sx } \\
\text { e.g., }\end{array}$ & \\
\hline Voltage & volt & $\begin{array}{r}x x \\
\text { e.g., }\end{array}$ & \\
\hline Current & Amp. & $\begin{array}{r}x x \\
\text { e.g., }\end{array}$ & \\
\hline
\end{tabular}




\subsection{Ground Transceiver Station}

The core of the APRS ground transceiver station (GTS), also known as i-Gate, is built by the RPi single board computer. It is programmed by using Python to control data processing, and then relays data to UTM Cloud via Internet. The flowchart of programs in RPi is shown in Figure 4.

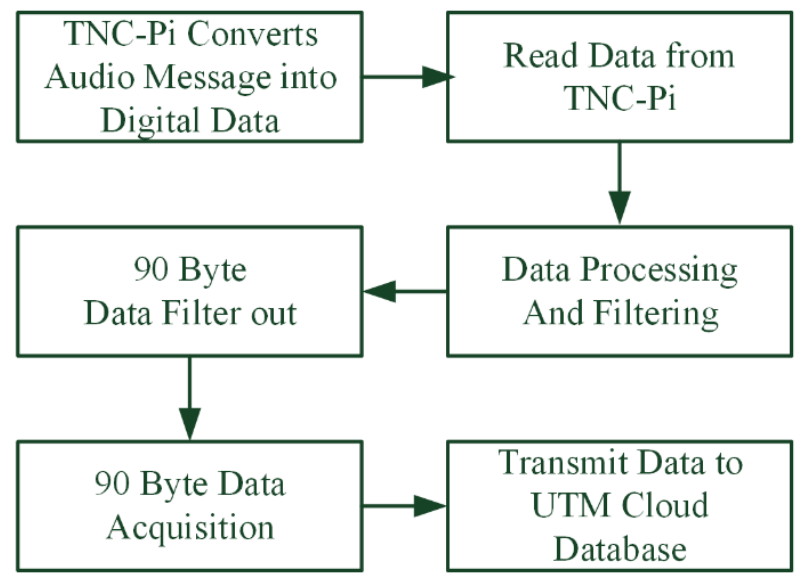

Figure 4. Flowchart of program in Raspberry Pi.

The APRS transmits data in audio message. Thus, the TCN-Pi recovers audio messages from the $\mathrm{RF}$ receiver back into digital data first, and then transmits digital data to RPi through the serial port as

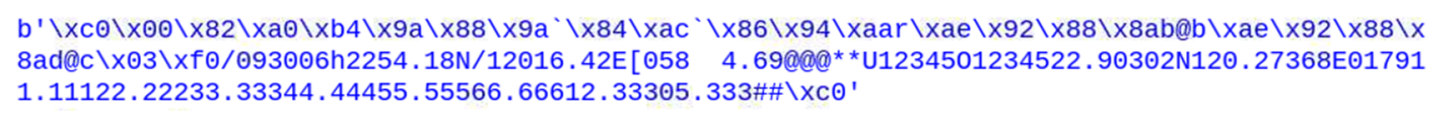

The packet format using a KISS protocol, a simple Host-to-TNC communication protocol, starts with the hexadecimal number $0 \times \mathrm{CC} 0$ and ends with $0 \times \mathrm{xC} 0$. As the RPi receives data from TNC-Pi, it proceeds to filter out the data of interest from the specific symbol code until the last $0 x \mathrm{C} 0$. In the APRS OBU, this symbol code is designed as "[" in the middle of the second line. The acquired data is ordered into a data string for post-processing as

058 12.03@@@**U1234501234522.90302N120.27368E017911.11122.22233.33344.44455.55566.66612.33

305. 333\#\#

This data string will be the typical ADS-B Like 90 Byte data for UTM surveillance. The data "058" and "12.03" indicate the transmitting sequence and LightAPRS voltage. Transmitting sequence just marks the data being sent. LightAPRS voltage notifies the user of OBU status. Then, it reads the 90 Byte surveillance data from heading “@@@**” to tailing "\#\#" for UTM. The final step will pass the received data using the POST method in HTTP protocol into the UTM database. The assigned format for POST method is as follows, and D1 to D6 represents the 6-DoF flight data.

\{'DroneId': 'U12345', 'operaterId': '012345', 'Latitude': 22.90302, 'Longitude': 120.27368

, 'Altitude': 179.0, 'D1': 11.111, 'D2': 22.222, 'D3': 33.333, 'D4': 44.444, 'D5': 55.555,

'D6': 66.666, 'V': 12.333, 'A': 5.333$\}$

\section{Results}

Test case 1:

This test aims to verify the reliability of collecting 90 byte surveillance data near the GTS site. The flight plan is to let the aircraft fly in four different directions to test the reliability of collecting data. With APRS OBU at 0.5 W RF power, the flight test uses the DJI M600 hexa-rotor UAV, as shown in 
Figure 5, to fly from CJCU campus to a nearby area. Since the flight control data cannot be acquired from the flight controller, the APRS OBU transmits the position data and the hard-code flight control data to UTM cloud in this test. The APRS OBU is mounted on the top of the UAV. In addition, LoRa OBU, another ADS-B like system, is also included for the test. The antenna of APRS GTS is fixed on the rooftop of a building on the CJCU campus as shown in Figure 6. From the UTM controller center, whenever the UAV takes off to fly, its position will be shown clearly on the UTM surveillance display as shown in Figure 7.

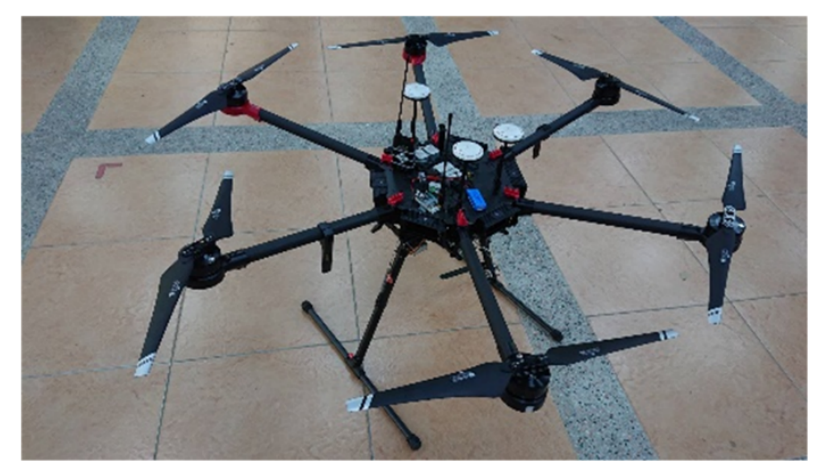

Figure 5. The hexa-rotor UAV for tests.

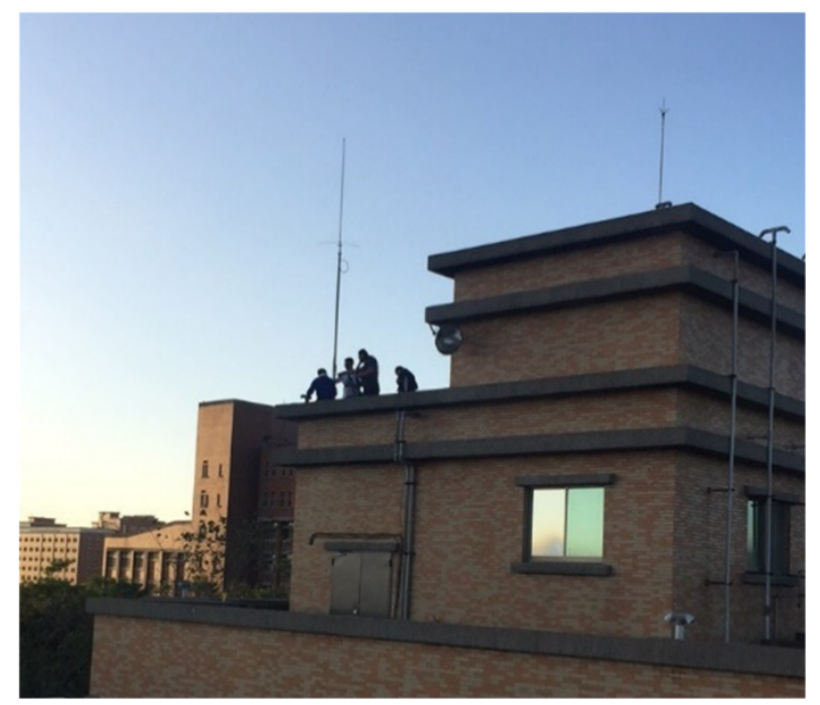

Figure 6. APRS GTS antenna at rooftop ( $42 \mathrm{~m}$ AGL) on campus.

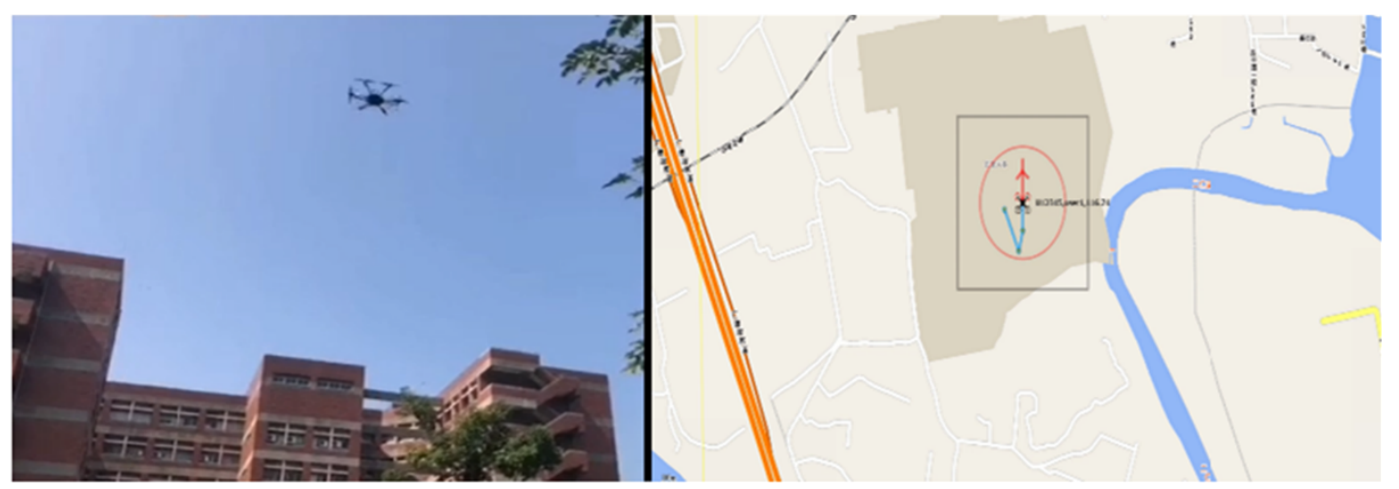

Figure 7. UTM surveillance display with flight test on campus. 
Figure 8 shows the flight test around CJCU campus with a flight track record of about $4.7 \mathrm{~km}$ at $55 \mathrm{~m}$ (AGL) altitude at 3-4 m/sec cruise speed. The maximum distance between the UAV and APRS GTS is about $1.4 \mathrm{~km}$. The APRS OBU reports 90 byte of surveillance data about every $10 \mathrm{~s}$. In this test, all of the received surveillance data in GTS have a complete 90 bytes. The test result shows that the APRS ADS-B like system works well under different UAV flight directions.

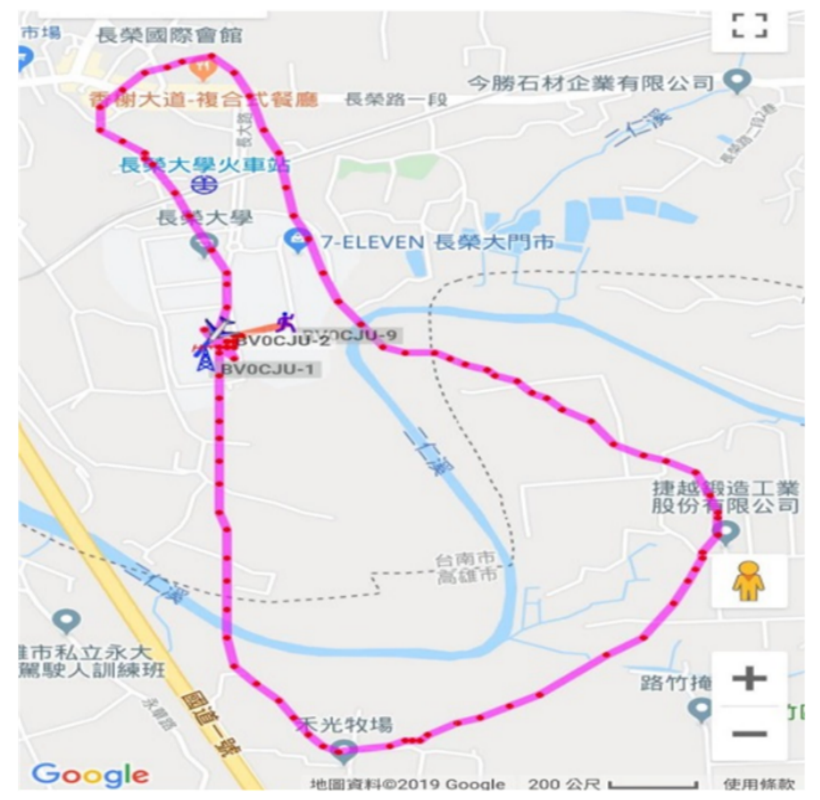

Figure 8. Flight surveillance test near CJCU campus.

To test the APRS ADS-B like coverage, a series of flight test are conducted from the near site about $4 \mathrm{~km}$ to the remote site about $40 \mathrm{~km}$ away from CJCU GTS. The DIY 550 quad-rotor UAV-equipped with GPS, Pixhawk FC, APRS OBU, and $433 \mathrm{MHz}$ telemetry-is adopted for these tests. The usage of $433 \mathrm{MHz}$ telemetry is for uploading the flight mission to Pixhawk. The right hand side in Figure 9 shows the 550 quad-rotor UAV, assembled by: $550 \mathrm{~mm}$ carbon airframe, 4108-390 kV motor, 30A ESC, 1555 propeller, and $6 \mathrm{~S} 25 \mathrm{C} 5800 \mathrm{mah}$ LiPo battery. The purpose for these tests was to examine: (1) the effective range of all communication devices; (2) complete 90 byte surveillance data; (3) data stability and continuity; (4) UAV attitude by 6 DoF.
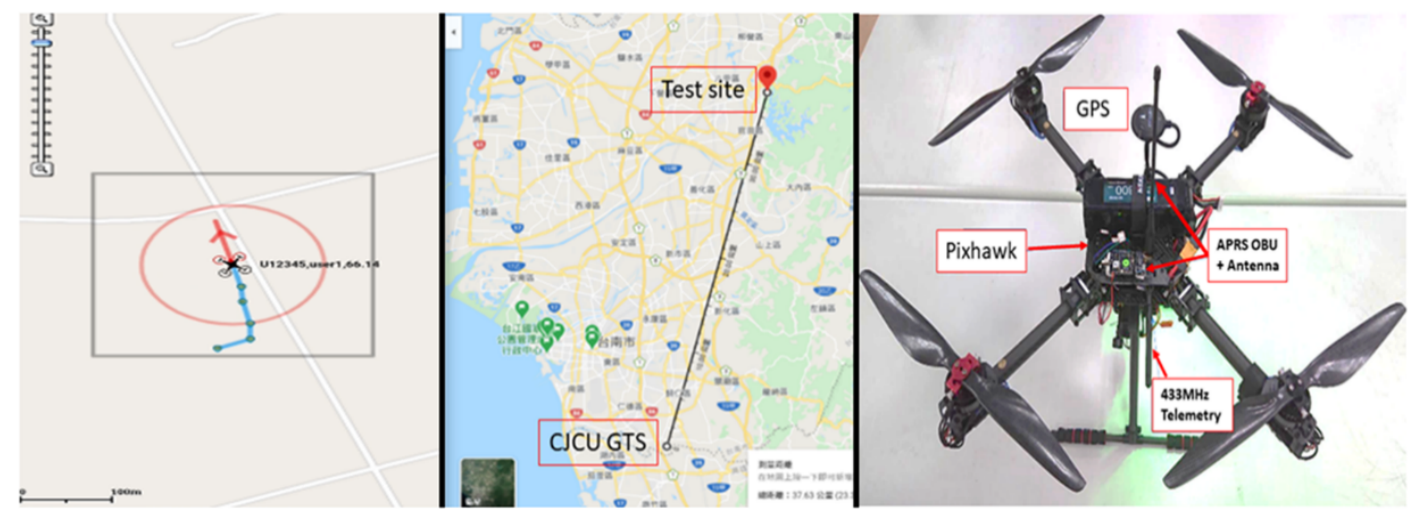

Figure 9. Remote test at $37.6 \mathrm{~km}$ away from CJCU GTS for APRS ADS-D like.

Test case 2:

In this test, the test site was located $37.63 \mathrm{~km}$ away from CJCU GTS with an altitude of $90 \mathrm{~m}$ above MSL. The UAV flew at an altitude of $184 \mathrm{~m}$ above MSL and circling a $800 \mathrm{~m}$ radius. In Figure 9, 
the UAV icon is displayed on the UTM surveillance system in the left hand side leaving six points behind as its past track.

The 90 byte surveillance data from APRS OBU are collected as follows (Supplementary Materials):

2020-02-21 18:14:53 028 4.83

@@@**U00005O0000523.23106N120.36976E0184

$1.28-0.710 .05-4.2-50.52 .624 .378 .53 \# \#$

The data begins with date, time, data sequence number 028 , and 4.83 for OBU voltage. The data starts from heading “@@@**”, UAV ID “U00005”, Pilot ID “O00005”, surveillance data, and tailing by "\#\#" to clearly distinguish from other APRS users. The real flight data is shown in Table 3. The UAV ID and Pilot ID are connecting to the management information system (MIS) from CAA [9].

Table 3. Real flight 90 Byte UTM surveillance data.

\begin{tabular}{cccc}
\hline Term & Data & Term & Data \\
\hline Date & $2020-02-21$ & $\mathrm{~V}_{\mathrm{x}}$ & $1.28 \mathrm{~m} / \mathrm{s}$ \\
Time & $18: 14: 53$ & $\mathrm{~V}_{\mathrm{y}}$ & $-0.71 \mathrm{~m} / \mathrm{s}$ \\
Sequence & 28 & $\mathrm{~V}_{\mathrm{z}}$ & $0.05 \mathrm{~m} / \mathrm{s}$ \\
Voltage & $4.83 \mathrm{~V}$ & Pitch & $-4.2^{\circ}$ \\
Heading & @@@** & Yaw & $-50.5^{\circ}$ \\
UAV ID & U00005 & Roll & $2.6^{\circ}$ \\
Pilot ID & O00005 & $\mathrm{V}$ & $24.37 \mathrm{~V}$ \\
Latitude & $23.23106 \mathrm{~N}$ & $\mathrm{I}$ & $8.53 \mathrm{~A}$ \\
Longitude & $120.36976 \mathrm{~N}$ & Tail & $\# \#$ \\
Altitude & $184 \mathrm{~m}$ & & \\
\hline
\end{tabular}

Figures 10-13 show the surveillance data in statistics in accordance with the data sequence number. Figure 10 shows the actual data receiving intervals. The varying intervals are due to a delay in fetching data from Pixhawk FC. The delay time according to radio traffic and congestion is minor in the tests. The interval is measured by the difference of receiving time for successive surveillance data in APRS GTS. The maximum fluctuation is about $4 \mathrm{~s}$, and is in the acceptable range for UTM operation. Figure 11 shows the UAV altitude tracking above MSL.

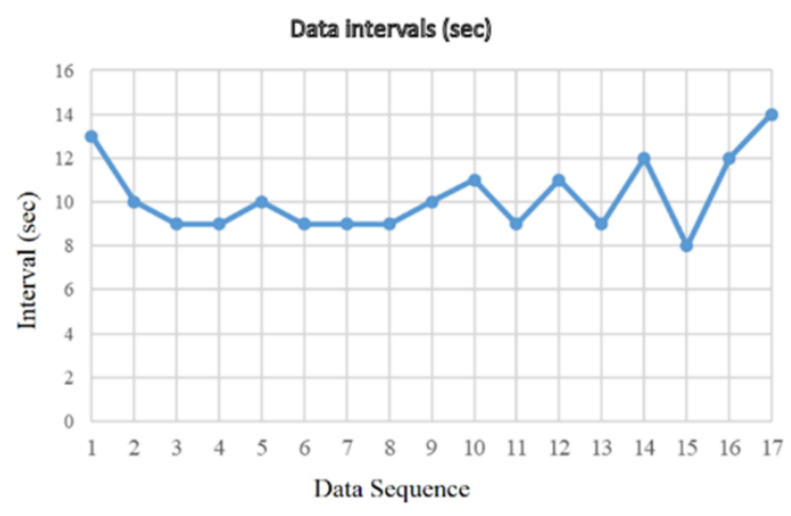

Figure 10. Intervals of surveillance data receiving. 


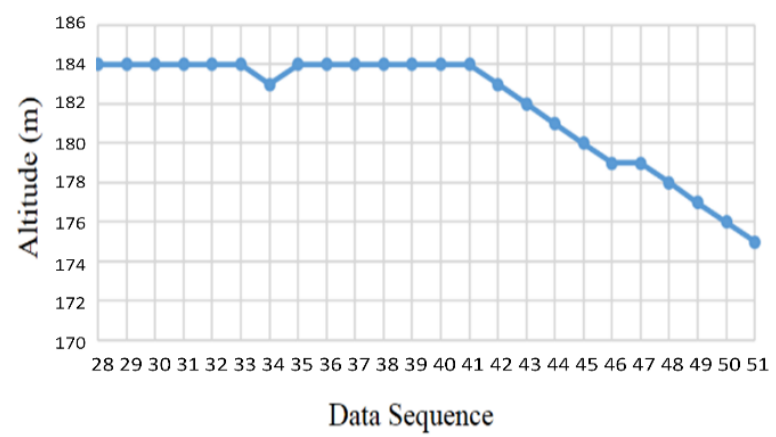

Figure 11. UAV altitude tracking.
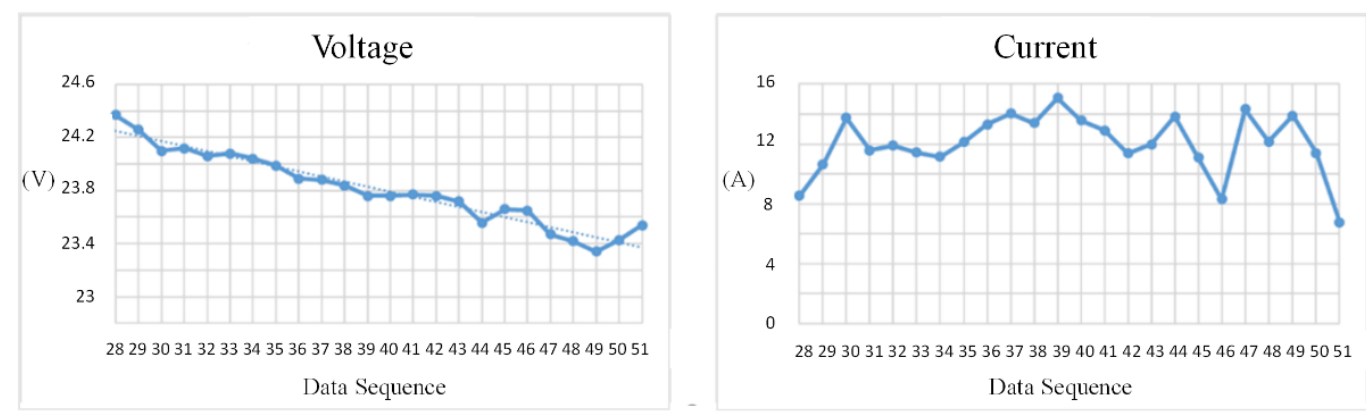

Figure 12. UAV voltage and current tracking.
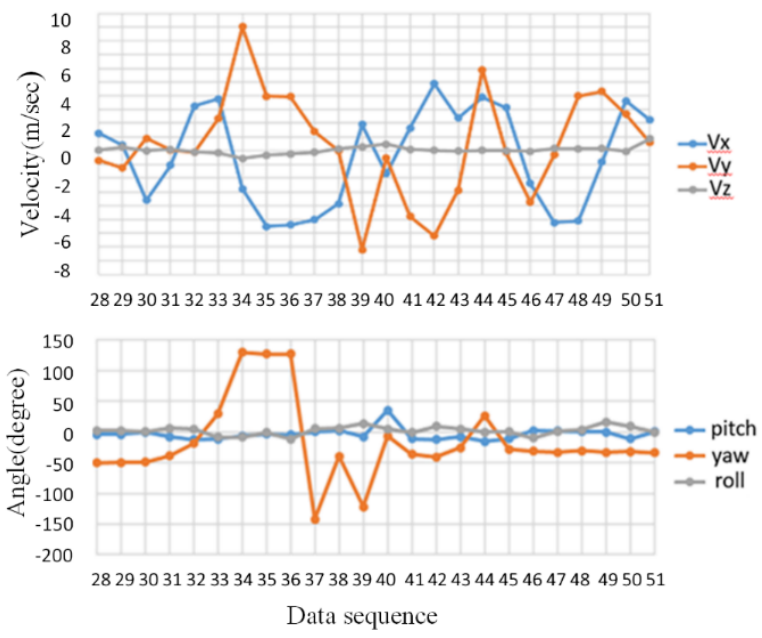

Figure 13. Surveillance data of 6-DoF UAV for test case 2.

Figure 12 shows the power supply status along the flight. It is quite useful to give an early warning to pilots if battery power supply is not sufficient at this point. Figure 13 shows the tracking of 6-DoF UAV during the flight. The UTM controller can check the UAV's status including position and 6-DoF flight data by using the 90 byte surveillance data from APRS OBU. From the yaw in Figure 13, it is obvious that the UAV have a right turn in flight according to data no. 36 and 37. Moreover, the flight altitude is held in flight since the value of $\mathrm{Vz}$ was kept around 0 .

Test case 3:

This test was conducted at the test site $4.4 \mathrm{~km}$ away from CJCU GTS. The UAV flew at an altitude of $184 \mathrm{~m}$ above MSL in a square flight path. Figure 14 shows the power current variation with respect to altitude change. It indicates clearly the high current change due to climbing. The current with respect to data no. 22 is about $0 \mathrm{~A}$ since the $\mathrm{UAV}$ is on the ground and ready to take off. Figure 15 shows the APRS OBU reporting positions and the in-flight UAV displays on the UTM surveillance 
system. Figure 16 shows the UAV surveillance data of 6-DoF UAV. The turning in the flight can be easily identified by observing the change of yaw angle. In addition, the NED-based velocities can be used to determine the flight direction. For example, the velocities of data no. 25 indicate the UAV flight direction is in southwest by south. In this test, the GTS receives all of the APRS OBU reporting surveillance data with the complete 90 bytes.
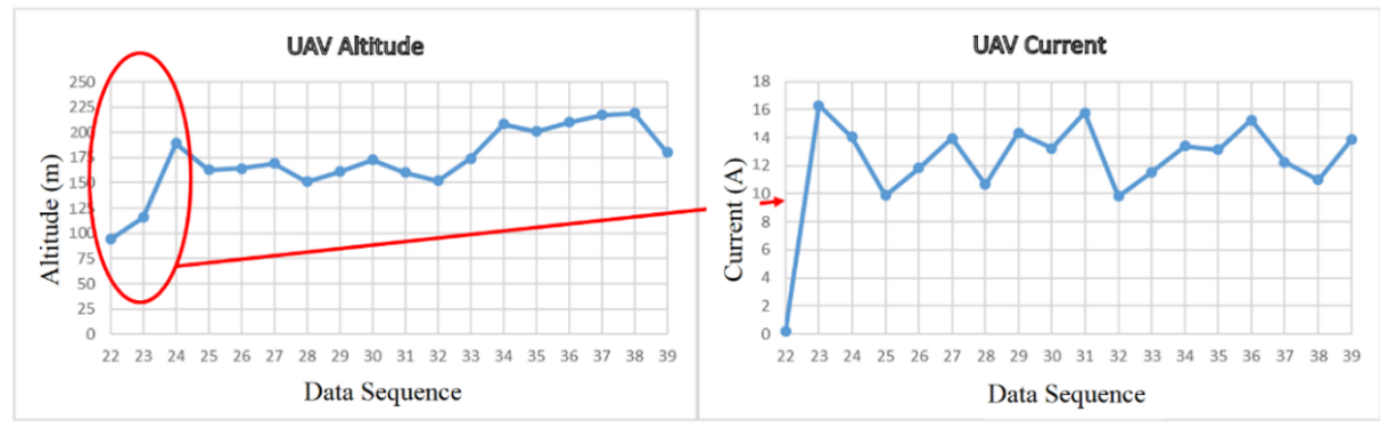

Figure 14. OBU surveillance data for cruise altitude and current.
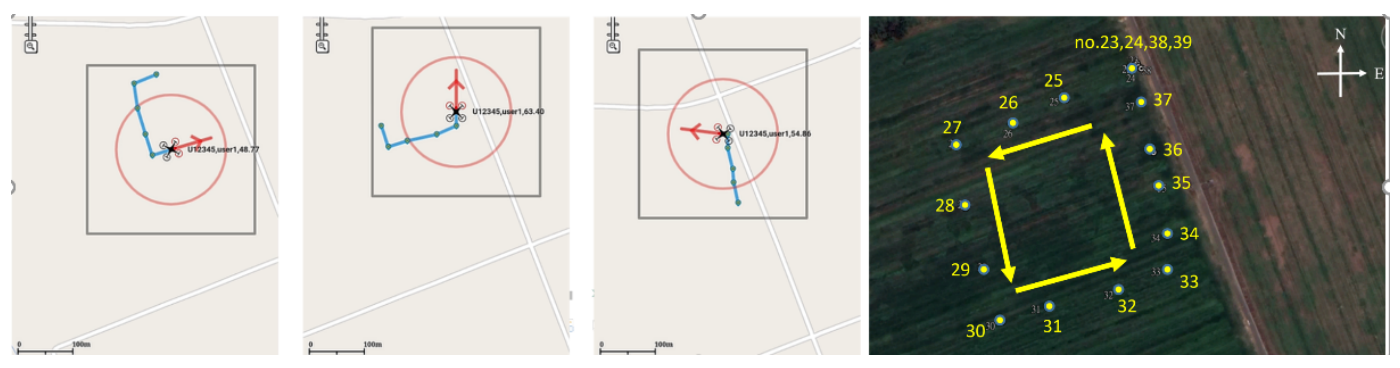

Figure 15. In-flight UAV displays on UTM surveillance system.

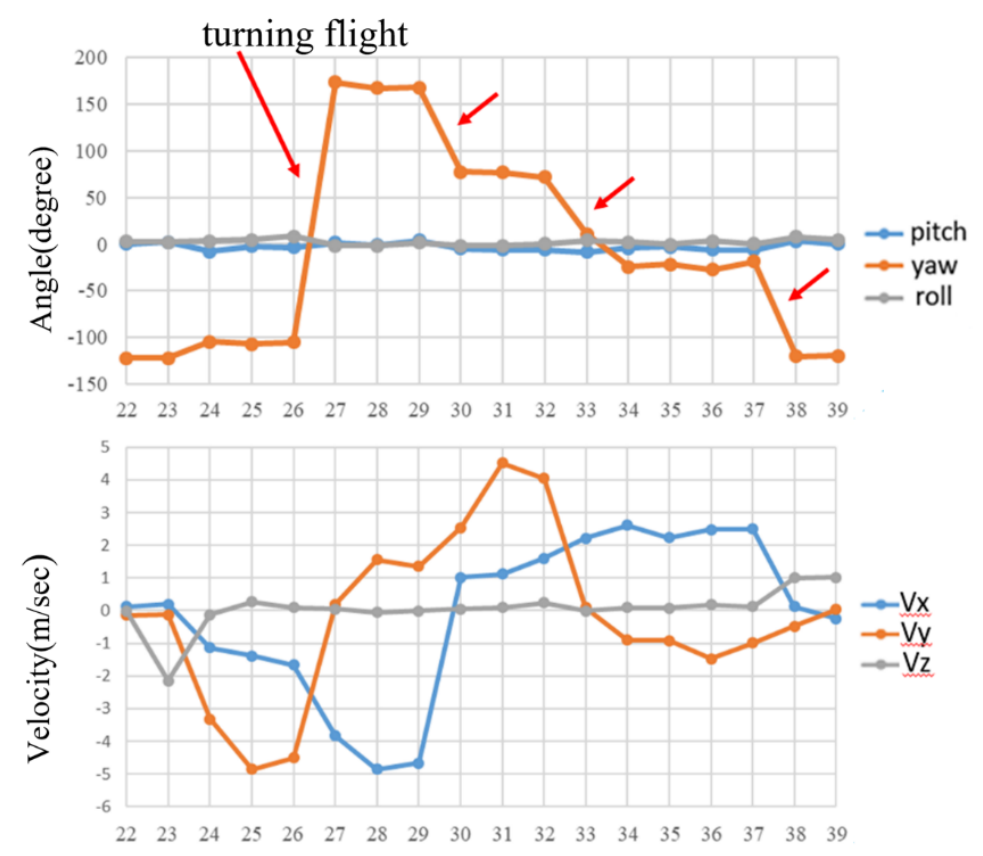

Data sequence

Figure 16. Surveillance data of UAV 6 DoF for test case 3.

The UAV attitude can be shown on UTM display during flight performance, these are data no. 36 and 37 in test case 2 in Figure 17. 


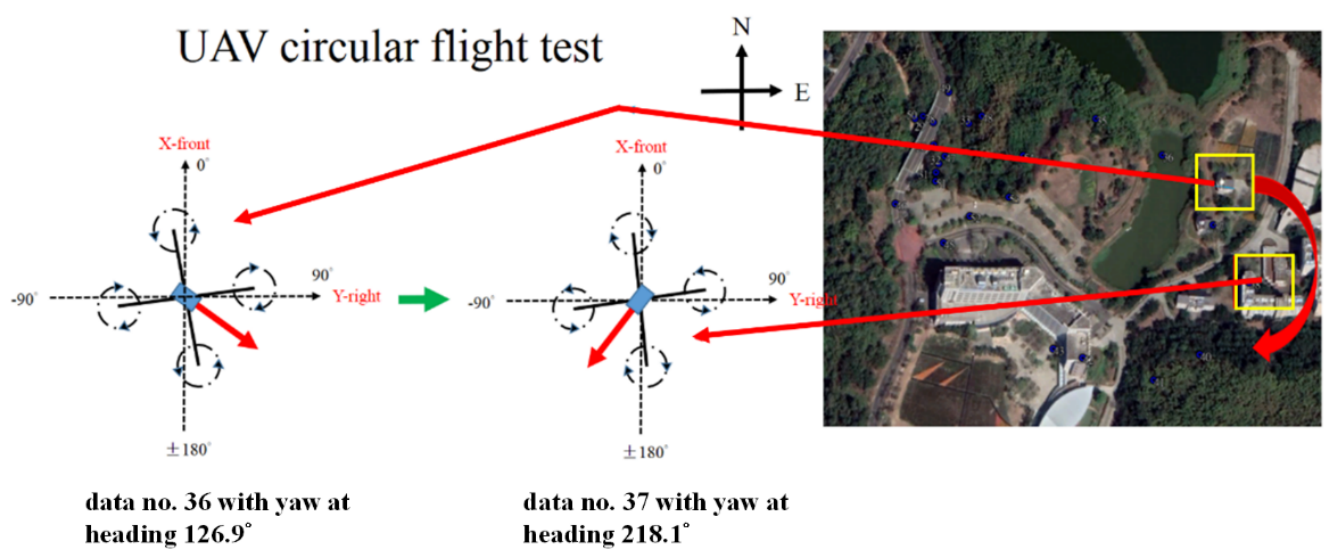

Figure 17. Flight data no. 36 and no. 37, UAV yaw turns from $126.9^{\circ}$ to $218.1^{\circ}$.

Using a different presentation, the UAV attitude can be displayed as a primary flight display (PFD) in Figure 18 while the UAV is turning. This is more convenient for observing the UAV's in-flight situation.

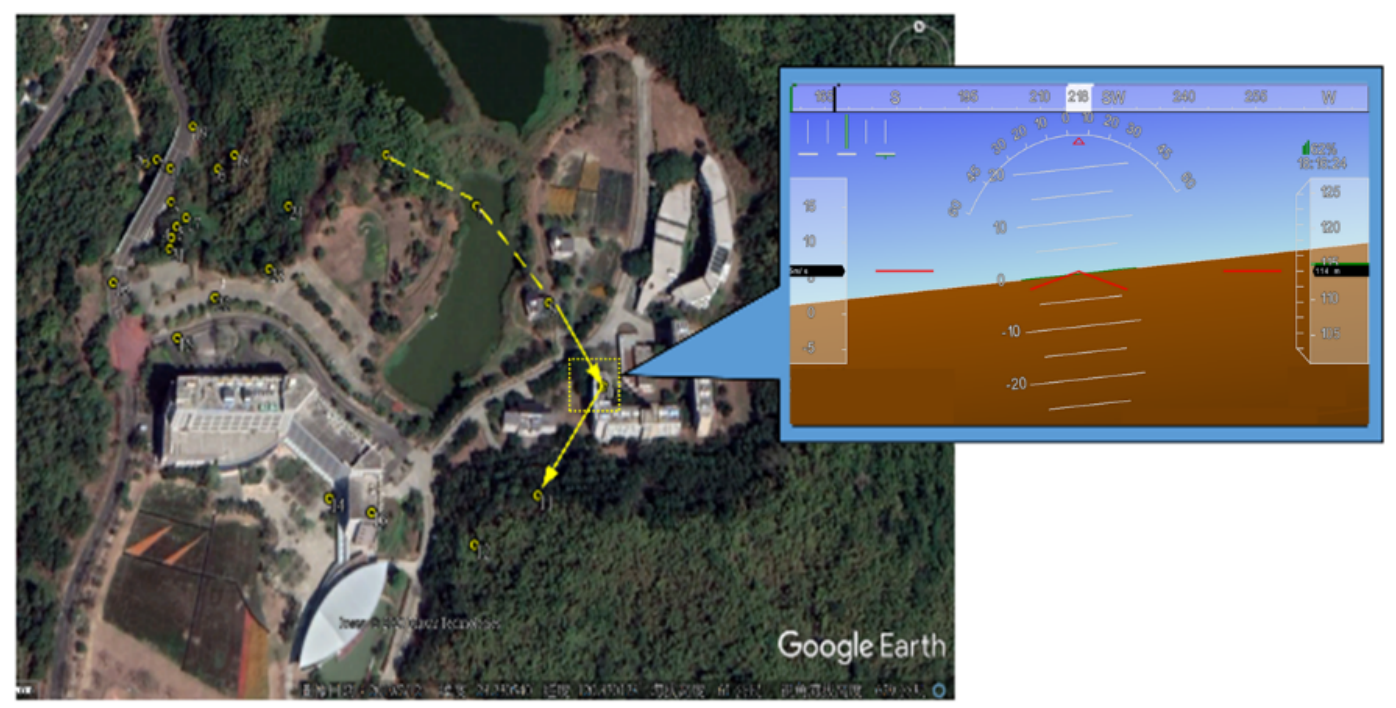

Figure 18. UTM surveillance display with checking UAV attitude.

\section{Discussion}

Through 19 flight tests, the CJCU GTS collected 1330 packets of data out of the 1331 transmitted from the APRS OBU. Each data packet has a complete 90 bytes for surveillance with position and 6 DoF to the UTM cloud. These tests are based on the APRS OBU with $0.5 \mathrm{~W}$ radio power and GTS using $6 \mathrm{dBi}$ antenna. From authors' experience, carefully adjusting the voice volume of RF receiver in GTS is an important step to reduce the rate of missing data. The rate of missing data is about $0.07 \%$ in these tests. It is concluded that the APRS ADS-B like system works well for UTM operation in transmission reliability and data continuity. Moreover, the APRS ADS-B like system collects 6-DoF data for UAV flying onto the UTM cloud. The big data will form the basis for further analysis and study on UAV flight operation quality assurance (FOQA).

Since the reporting interval of the APRS OBU is around $10 \mathrm{~s}$, the 90 byte surveillance data reveals conditions of the UAV in-flight quickly. They are valuable not only for identifying the flight direction, position, operation, etc., but also for predicting the health of a UAV in-flight using the surveillance 
data stored in the UTM cloud. For example, the excessive drop in voltage indicates damage on the UAV battery.

The UTM system can offer UAV service providers with in-flight data as redundancy if any malfunction or lost contact during the flight occurs. Under request, the UTM system can offer the pilots or UAV service providers to back up the flight data.

\section{Conclusions}

In UTM development, the proper OBU for surveillance data reporting is the key device to support the complete UAV surveillance. This study develops a APRS OBU, based on available LightAPRS modules, capable of reporting the GPS position and UAV 6 DoF acquired from Pixhawk FC. Moreover, the APRS GTS is also developed to relay the 90 byte surveillance data onto the UTM cloud. By conducting 19 flight tests at different sites from $4 \mathrm{~km}$ to $40 \mathrm{~km}$ away from the CJCU GTS, the GTS collected 1330 packets of data out of 1331 reported from the APRS OBU. Each received data packet in the GTS has a complete 90 bytes for surveillance with position and UAV 6 DoF. The test results verify the availability of the APRS OBU to successfully transmit surveillance data to the UTM Cloud with satisfactory continuity and reliability. Furthermore, the APRS GTS deployment, with a low rate of missing data, covers a $40 \mathrm{~km}$ radius at a specific frequency $144.61 \mathrm{MHz}$.

Based on the test results mentioned above, the developed APRS ADS-B like system is capable of working in the Tainan City UAV Integrated Pilot Program (IPP), and supports full surveillance for rotor-wing UAVs flying $30 \mathrm{~m}$ above ground level to fixed-wing UAVs flying in high altitudes. According to APRS ADS-B like functional capability, its altitude coverage can be extended to 20,000 feet or higher. By distinguishing UAVs from manned aircraft, the UTM can offer services to rotor-wing UAVs flying below 400 feet or fixed-wing UAVs flying higher.

The development of hierarchical UTM [4] proposes four candidates of ADS-B like [6] on-board unit for UAVs to report data periodically to UTM cloud. Complete flight surveillance can be accomplished with ADS-B like supports. It is the most important progress for UAV flight under effective UTM operation [15].

\section{Patents}

Taiwanese invention patent, "ADS-B Like On-Board Unit", was submitted with p-8499 on 26 August 2019.

Supplementary Materials: The following are available online at: https://reurl.cc/0orllM.

Author Contributions: For this research work, C.E.L. proposed the system concept and organized the experiment for verification, he also wrote and edited this paper for Aerospace. Y.-H.L. carried out system design and implementation to prepare hardware for tests. H.-C.C. conducted hardware fabrication and software programming. All authors have read and agreed to the published version of the manuscript.

Funding: This is an academic research project supporting from the Ministry of Science and Technology under contract MOST 108-2622-E-309-001-CC1.

Acknowledgments: This UTM developing project is supported by Ministry of Science and Technology (MOST) under contract MOST 108-2622-E-309-001-CC1 in cooperation with Chung-Hua Telecommunication (CHT) for system development.

Conflicts of Interest: The authors declare there is no conflict to any institution or organization for this research results.

\section{References}

1. ICAO. Air Traffic Management Doc 4444, 16th ed.; ICAO: Montreal, QC, Canada, 2016; ISBN 978-92-9258-081-0.

2. Kopardekar, P.; Rios, J.; Prevot, T.; Johnson, M.; Jung, J.; Robinson, J.E. Unmanned Aircraft System Traffic Management (UTM) Concept of Operation. In Proceedings of the 16th AIAA Aviation Technology, Integration and Operation Conference ATIO, Washington, DC, USA, 13-17 June 2016. 
3. Kamienski, J.; Semanek, J. ATC Perspectives of UAS Integration in Controlled Airspace. In Proceedings of the 6th International Conference on Applied Human Factors and Ergonomics (AHFE) and the Affiliated Conference, Las Vegas, NV, USA, 26-30 July 2015.

4. Lin, C.E.; Chen, T.P.; Shao, P.C.; Lai, Y.C.; Chen, T.C. Prototype Hierarchical UAV Traffic Management System in Taiwan. In Proceedings of the Integrated Communication, Navigation and Surveillance ICNS, Dulles Airport, Washington, DC, USA, 9-11 April 2019.

5. Federal Aviation Administration. TCAS II V7.1 Introduction; US Department of Transportation: Washington, DC, USA, 2011.

6. Lin, C.E.; Hsieh, C.S.; Li, C.C.; Shao, P.C.; Lin, Y.H.; Yeh, Y.C. An ADS-B Like Communication for UTM. In Proceedings of the Integrated Communication, Navigation and Surveillance ICNS, Dulles Airport, Washington, DC, USA, 9-11 April 2019.

7. Lin, C.E.; Hung, T.W.; Chen, H.Y. TCAS Algorithm for Generation Aviation on ADS-B. Proc. Inst. Mech. Eng. Part G J. Aerosp. Eng. 2016, 230, 1569-1591. [CrossRef]

8. Kerczewski, R.J.; Apaza, R.D.; Downey, A.N.; Wang, J. Assessing C2 Communications for UAS Traffic Management. In Proceedings of the IEEE/AIAA Integrated Communication, Surveillance and Navigation Conference (ICNS), Herndon, VA, USA, 10-12 April 2018.

9. Civil Aeronautical Administration (CAA). Taiwan, Civil Aviation Act, UAS Chapter. Available online: https://www.caa.gov.tw/en/content/index.asp?sno=325 (accessed on 25 April 2018).

10. Lin, C.E.; Lee, C.J. ADS-R Conflict Detection and Resolution Algorithm for General Aviation in Low Altitude Flights. J. Aeronaut. Astronaut. Aviat. 2015, 48, 111-122. [CrossRef]

11. Lin, C.E.; Shao, P.C.; Li, C.C.; Chen, T.S.; Lai, Y.C.; Yeh, Y.C. Development of Hierarchical UAS Traffic Management (UTM) in Taiwan. J. Phys. Conf. Ser. 2020, 1509, 1-11. [CrossRef]

12. Orrell, G.L.; Chen, A.; Reynolds, C.J. Small unmanned aircraft system (sUAS) automatic dependent surveillance-broadcast (ADS-B) like surveillance concept of operations: A path forward for small UAS surveillance. In Proceedings of the 2017 IEEE/AIAA 36th Digital Avionics Systems Conference (DASC), St. Petersburg, FL, USA, 17-21 September 2017. [CrossRef]

13. Ramsey, C. The Case for Low Power ADS-B for Drones. Available online: https://uavionix.com/blog/thecase-for-low-power-ads-b/ (accessed on 20 May 2020).

14. Scardina, J. Overview of the FAA ADS-B Link Decision. In Proceedings of the U.S. Department of Transportation, Federal Aviation Administration Fifth Meeting, Saint Petersburg, Russia, 19-23 September 2002.

15. Lin, C.E.; Shao, P.-C.; Lin, Y.-Y. System Operation of Regional UTM in Taiwan. Aerospace 2020, 7, 65. [CrossRef]

16. LightAPRS Tracker. Available online: http://qrp-labs.com/lightaprs (accessed on 20 October 2019).

17. Pixhawk Overview. Available online: https://ardupilot.org/copter/docs/common-pixhawk-overview.html (accessed on 20 October 2019).

18. TNC-X for Raspberry Pi. Available online: https://tnc-x.com/TNCPi.htm (accessed on 20 October 2019).

19. AX.25 Link Access Protocol for Amateur Packet Radio. Available online: http://www.tapr.org/pdf/AX25.2.2. pdf (accessed on 20 October 2019).

20. MAVLink Interface. Available online: https://ardupilot.org/dev/docs/mavlink-commands.html (accessed on 20 October 2019).

(C) 2020 by the authors. Licensee MDPI, Basel, Switzerland. This article is an open access article distributed under the terms and conditions of the Creative Commons Attribution (CC BY) license (http://creativecommons.org/licenses/by/4.0/). 